\title{
BENEFICIOS DEL APRENDIZAJE COOPERATIVO EN LAS ÁREAS TRONCALES DE PRIMARIA: UNA REVISIÓN DE LA LITERATURA CIENTÍFICA
}

\section{BENEFITS OF COOPERATIVE LEARNING IN THE MAIN AREAS OF PRIMARY: A REVIEW OF THE SCIENTIFIC LITERATURE}

\author{
Salvador Boix Vilella \\ salvador.boix@ui1.es \\ Facultad de Humanidades y Ciencias Sociales. Universidad Isabel I (España) \\ Nazareth Ortega Rodríguez \\ nazareth.ortega@educa.madrid.org \\ CEIP. Doctor Severo Ochoa de Getafe (España)
}

Recibido: $10 / 12 / 2018$

Aceptado: 04/06/2020

\begin{abstract}
Resumen:
El aprendizaje cooperativo se viene utilizando en educación primaria como alternativa a métodos educativos tradicionales y competitivos. El objetivo de este estudio es mostrar los beneficios académicos, afectivos y sociales del aprendizaje cooperativo en las áreas troncales de la educación primaria (lengua castellana, matemáticas, ciencias naturales, ciencias sociales e inglés). Para localizar los trabajos se accede a las bases de datos: Dialnet, ERIC, ScienDirect y Scielo. Finalmente, se analizan un total de 14 trabajos. Se destacan los beneficios afectivos y sociales por encima de los académicos, destacando el aumento de las habilidades sociales y la mejora del clima del aula. Como conclusión, el aprendizaje cooperativo genera beneficios académicos que repercuten especialmente en el modo de acceder al conocimiento y no tanto en sus resultados. Además, parece mejorar la inclusión.
\end{abstract}

Palabras clave: aprendizaje cooperativo, ciencias naturales, ciencias sociales, lengua, matemáticas.

\footnotetext{
Abstract:

Cooperative learning is being used in primary education as an alternative to traditional and competitive educational methods. The objective of this study is to show the academic, affective and social benefits of cooperative learning in the main areas of primary education (Spanish language, mathematics, natural sciences, social sciences and English). All works are available at
} 
the databases: Dialnet, ERIC, ScienDirect and Scielo. Finally, a total of 14 papers are analyzed. The affective and social benefits are stand out over academics, highlighting the increase in social skills and the improvement of the classroom climate. In conclusion, cooperative learning generates academic benefits that have a particular impact on how knowledge is accessed and not so much on its results. In addition, it appears to improve the inclusion.

Keywords: cooperative learning, natural sciences, social sciences, language, mathematics.

\section{Introducción}

Las sociedades industrializadas del siglo XXI vienen experimentando constantes cambios que repercuten directa o indirectamente en el ámbito educativo de cada país (Chamseddine, 2018). En el caso de España, parte de la ciudadanía solicita a los centros escolares y a las administraciones públicas la incorporación de nuevas metodologías educativas que dejen atrás la visión tradicional, competitiva e individualista del aprendizaje (Pujolàs, 2012). Sin embargo, tanto la Ley Orgánica para la Mejora de la Calidad Educativa (LOMCE, 2013) como la Orden ECD65/2015 por la que se describen las relaciones entre las competencias, los contenidos y los criterios de evaluación de la educación primaria ya contemplan en su articulado la necesidad de una práctica docente con metodologías más motivadoras que atiendan a las actuales demandas del ciudadano y mejoren la calidad educativa.

En este contexto de cambios legislativos y organizativos, los resultados de evaluaciones externas internacionales junto con las evaluaciones nacionales, derivadas de la legislación vigente, permiten conocer las fortalezas y las debilidades de las diferentes etapas con el fin de mejorar el sistema educativo español. En el Estudio Internacional de Tendencias en Matemáticas y Ciencias (TIMSS), los resultados presentados por el Instituto Nacional de Evaluación Educativa (INEE, 2016) sitúan, en el 2015, al alumnado español de cuarto de primaria ligeramente por debajo de la media de puntuaciones obtenidas por el conjunto de la Unión Europea (UE) y de los países de la Organización para la Cooperación y el Desarrollo Económicos (OCDE). En el caso del Estudio Internacional de Progreso en Comprensión Lectora (PIRLS) también centrado en cuarto de primaria, las puntuaciones españolas del 2016 son inferiores a la media de la UE y la OCDE, según los datos obtenidos a partir del informe de resultados publicados por el INEE (2017). Las pruebas internas de evaluación en la etapa de primaria vienen marcadas inicialmente en la Ley Orgánica de Educación (LOE, 2006) y modificadas posteriormente por la LOMCE (2013). Cada comunidad autónoma es la encargada de desarrollar una evaluación de carácter diagnóstico al finalizar tercero y sexto de primaria. En función del nivel en el que se aplican, se abordan más o menos competencias aunque en ambas pruebas se priorizan los contenidos de las áreas troncales. Al finalizar tercero, se evalúa la competencia en comunicación lingüística (mediante pruebas de comprensión oral, comprensión escrita y expresión escrita) y la competencia matemática y en sexto también se incluyen las competencias básicas en ciencia y tecnología. Todos estos resultados internacionales y nacionales, existentes en la etapa de primaria, junto con los obtenidos en las pruebas PISA en secundaria han contribuido a avivar el debate social y político sobre la calidad del sistema educativo español no universitario (González-Mayorga, Vidal y Vieira, 2017).

Entre las diferentes metodologías educativas que se vienen empleando actualmente en los centros escolares, el aprendizaje cooperativo ha despertado el interés de numerosos investigadores y maestros que han empezado a aplicarlo en sus aulas de una manera habitual como consecuencia de los buenos resultados que se le atribuyen (Ribosa y Durán, 2017; Suárez, 2013). Pero su utilización va más allá de las aulas; existen publicaciones que señalan que el aprendizaje cooperativo en las horas de recreo genera una mayor cohesión grupal, favorece la 
formación de nuevas relaciones y con ello se reduce el número de conflictos entre el alumnado (Mega y Liesa, 2015; Vázquez, Liesa y Lozano, 2017). Además, su utilización se considera adecuada y beneficiosa para todas las etapas educativas, tanto universitarias como no universitarias (Iglesias y López, 2014; Pegalajar, 2018; Santos-Rego, Lorenzo-Moledo y PriegueCaamaño, 2009).

La idea de aprendizaje cooperativo no es reciente ya que aparece a principios del siglo XIX en una escuela lancasteriana, ubicada en Nueva York (Lobato, 1997). Aunque su utilización no fue realmente significativa hasta mediados del siglo XX, que pasó a utilizarse como una estrategia de organización, por parte de los docentes, con el objetivo de conseguir la integración escolar (Johnson y Johnson, 1994; Slavin, 1980). El aprendizaje cooperativo se puede definir como un trabajo en grupo que se estructura de manera cuidadosa para que todos los individuos interactúen, intercambien información, trabajen de manera coordinada y puedan ser evaluados de forma individual por su tarea (Fathman y Kessler, 1993). Otra definición más reciente lo considera un modelo pedagógico en el que profesor y estudiantes actúan como co-aprendices (Fernández-Río, 2014). Se debe tener en cuenta que no cualquier agrupación o equipo de alumnos realiza su proceso de aprendizaje de manera efectiva, firme o eficiente por el mero hecho de realizar sus tareas en grupo, sino que para que esto se produzca los miembros del grupo necesitan educarse en ello (Cassany, 2009).

En relación con la estructura organizativa del aprendizaje cooperativo, los autores Kagan y Kagan (1994) defienden la interacción simultánea, la igualdad de posibilidades de participación dentro del grupo de estudiantes y la interdependencia positiva para mejorar el clima del aula y favorecer así la responsabilidad individual. La idea de cooperación entre estudiantes incide en que debe existir un sentimiento de recompensa tras su aprendizaje, como fruto de la ayuda recibida en el equipo, mejorando con ello las relaciones humanas (DeVries y Edwards, 1974). Dentro del aprendizaje cooperativo, aparece el modelo teórico de instrucción compleja de Cohen (1994) que aporta el concepto de estatus preconcebido con el que cada integrante entra al grupo. Para que estas variables personales, sociales y/o culturales no afecten al correcto desarrollo del grupo es primordial tratar de modificar prejuicios, preparar a los alumnos para la cooperación y dar a cada miembro del grupo el rol o tarea que precisa (Cohen, 1994). En definitiva, se persigue trabajar para lograr la inclusión de todos (Lata y Castro, 2016).

Pujolàs (2009) contempla diferentes perspectivas (motivacional, cohesión social, cognitiva, práctica y organización del aula) que explicarían los efectos del aprendizaje cooperativo sobre el rendimiento del alumno. Lo que parece evidente es que el aprendizaje cooperativo es una realidad en las aulas españolas y tiene una presencia importante en la etapa de primaria ya que se le presuponen resultados positivos en el ámbito social, afectivo y académico (Carrasco, Alarcón y Trianes, 2015).

En función de los antecedentes expuestos, el presente trabajo de revisión se plantea dos objetivos: (a) mostrar los beneficios académicos, afectivos y sociales del aprendizaje cooperativo en las áreas troncales de la educación primaria (lengua castellana y literatura, matemáticas, ciencias de la naturaleza, ciencias sociales e inglés); (b) sugerir futuras líneas de actuación relativas al aprendizaje cooperativo en las áreas troncales de la etapa de primaria.

\section{Metodología.}

\subsection{Procedimiento de búsqueda bibliográfica}

El primer paso de este trabajo de revisión bibliográfica consiste en seleccionar aquellos trabajos que contienen información relevante para dar respuesta a los objetivos propuestos. El acceso a estos documentos se realiza a través de bases de datos científicas que incluyen investigaciones 
sobre ciencias sociales y educación. Las bases de datos seleccionadas para abordar el estado actual del aprendizaje cooperativo en primaria han sido: ScienceDirect, Dialnet, Scielo y ERIC. Todas ellas permiten el acceso a trabajos con el rigor científico necesario y a texto completo en su gran mayoría.

En la búsqueda se han introducido en todas las bases de datos los descriptores: aprendizaje cooperativo, aprendizaje cooperativo AND primaria, aprendizaje cooperativo AND matemáticas, aprendizaje cooperativo AND lengua, aprendizaje cooperativo AND inglés y aprendizaje cooperativo AND ciencias. Todos estos descriptores también se han empleado en esas mismas bases de datos traducidos al inglés.

\subsection{Criterios de inclusión y exclusión}

Una vez localizados los trabajos científicos, se aplican una serie de criterios de inclusión y de exclusión. La Tabla 1 muestra los criterios empleados con el fin de acotar la búsqueda inicial de trabajos y dar una respuesta precisa a los objetivos propuestos.

\begin{tabular}{|c|c|}
\hline Criterios de inclusión & Criterios de exclusión \\
\hline $\begin{array}{l}\text { - Trabajos que utilizan el término aprendizaje } \\
\text { cooperativo en el título, resumen y/o palabras } \\
\text { clave. }\end{array}$ & $\begin{array}{l}\text {-Trabajos que no abordan el aprendizaje } \\
\text { cooperativo como eje fundamental de la } \\
\text { investigación. }\end{array}$ \\
\hline -Artículos con texto completo. & $\begin{array}{l}\text { - Trabajos escritos en forma de resumen o } \\
\text { comunicación corta. }\end{array}$ \\
\hline $\begin{array}{l}\text {-Trabajos centrados en alumnos y profesores en } \\
\text { activo de la etapa de educación primaria. }\end{array}$ & $\begin{array}{l}\text { - Artículos centrados en alumnos y profesores en } \\
\text { activo de las etapas de: infantil, secundaria, } \\
\text { bachillerato y/o universitaria. }\end{array}$ \\
\hline $\begin{array}{l}\text {-Publicaciones comprendidas entre la fecha de } \\
\text { implantación de la LOMCE (enero 2014) hasta } \\
\text { diciembre de } 2018 .\end{array}$ & $\begin{array}{l}\text { - Trabajos publicados antes del año } 2014 \text { para } \\
\text { conocer los últimos avances en la cuestión tras la } \\
\text { LOMCE. }\end{array}$ \\
\hline dos on อcnวñol & $\begin{array}{l}\text { - Publicaciones en idiomas diferentes al español e } \\
\text { inglés. }\end{array}$ \\
\hline $\begin{array}{l}\text {-Artículos centrados en la realidad del } \\
\text { aprendizaje cooperativo en el sistema educativo } \\
\text { español (público, concertado y privado). }\end{array}$ & $\begin{array}{l}\text { - Trabajos que no aborden el aprendizaje } \\
\text { cooperativo desde la realidad española. }\end{array}$ \\
\hline $\begin{array}{l}\text {-Trabajos que relacionen el aprendizaje } \\
\text { cooperativo con alguna de las áreas troncales } \\
\text { (matemáticas, lengua castellana, ciencias } \\
\text { sociales, ciencias naturales e inglés) o en la } \\
\text { globalidad de primaria. }\end{array}$ & $\begin{array}{l}\text { - Trabajos que se centran de manera concreta en } \\
\text { las áreas de: educación física, educación artística, } \\
\text { educación musical, lenguas autonómicas y/o } \\
\text { religión. }\end{array}$ \\
\hline $\begin{array}{l}\text {-Artículos cuyos resultados han sido } \\
\text { rigurosamente obtenidos, con unos criterios de } \\
\text { sistematicidad y analizados de manera } \\
\text { conveniente como demanda el método } \\
\text { científico. }\end{array}$ & $\begin{array}{l}\text { - Trabajos sin el suficiente rigor científico que } \\
\text { abordan experiencias con un propósito } \\
\text { principalmente divulgativo. }\end{array}$ \\
\hline
\end{tabular}

Tabla 1. Criterios de inclusión y criterios de exclusión

\section{Resultados}

Tras la búsqueda en las diferentes bases de datos seleccionadas, la Tabla 2 muestra el número total de trabajos, con texto completo y publicados entre el 1 de enero de 2014 y el 31 de diciembre de 2018. Además, se muestra el número de estudios en función del idioma de las palabras clave utilizadas, español e inglés. 


\begin{tabular}{|c|c|c|c|c|c|c|c|c|c|c|}
\hline & \multicolumn{2}{|c|}{ ScienceDirect } & \multicolumn{2}{|c|}{ Dialnet } & \multicolumn{2}{|c|}{ ERIC } & \multicolumn{2}{|c|}{ Scielo } & \multicolumn{2}{|c|}{ Total } \\
\hline & esp. & inglés & esp. & inglés & esp. & inglés & esp. & inglés & esp. & inglés \\
\hline $\begin{array}{l}\text { Aprendizaje } \\
\text { cooperativo }\end{array}$ & 137 & 19320 & 1090 & 840 & 1 & 3968 & 71 & 121 & 1299 & 24249 \\
\hline $\begin{array}{l}\text { Aprendizaje } \\
\text { cooperativo } \\
\text { AND primaria }\end{array}$ & 51 & 9472 & 179 & 104 & - & 231 & 8 & 9 & 238 & 9816 \\
\hline $\begin{array}{l}\text { Aprendizaje } \\
\text { cooperativo } \\
\text { AND } \\
\text { matemáticas }\end{array}$ & 27 & 4692 & 48 & 38 & - & 437 & 4 & 8 & 79 & 5117 \\
\hline $\begin{array}{l}\text { Aprendizaje } \\
\text { cooperativo } \\
\text { AND lengua }\end{array}$ & 29 & 6509 & 100 & 90 & - & 691 & 8 & 7 & 137 & 7297 \\
\hline $\begin{array}{l}\text { Aprendizaje } \\
\text { cooperativo } \\
\text { AND ciencias }\end{array}$ & 80 & 12732 & 50 & 122 & - & 954 & 4 & 18 & 134 & 13826 \\
\hline $\begin{array}{l}\text { Aprendizaje } \\
\text { cooperativo } \\
\text { AND inglés }\end{array}$ & 20 & 3257 & 68 & 45 & 1 & 455 & 7 & 11 & 96 & 3768 \\
\hline
\end{tabular}

Tabla 2. Palabras clave y número de artículos encontrados entre 2014 y 2018 a texto completo.

Tras la aplicación de los criterios de inclusión y exclusión, 14 trabajos fueron analizados. En la Tabla 3, se muestran todos los estudios incluidos en esta revisión. En ella, se recogen los autores de cada trabajo seleccionado, el diseño del mismo, los participantes, el área o áreas en las que se ha centrado el estudio y los resultados más importantes encontrados.

\begin{tabular}{|c|c|c|c|}
\hline Estudio & Diseño & Participantes/áreas & Resultados \\
\hline $\begin{array}{l}\text { Gallach y } \\
\text { Catalán (2014) }\end{array}$ & Descriptivo & $\begin{array}{l}\text { Alumnado de } 40 \text { de } \\
\text { primaria ( } \mathrm{n}= \\
\text { desconocido, no se } \\
\text { incluye el total). Se } \\
\text { centra en las áreas de } \\
\text { lengua castellana, } \\
\text { ciencias sociales y } \\
\text { ciencias naturales. }\end{array}$ & $\begin{array}{l}\text { Mejora la relación entre la } \\
\text { diversidad de grupos del aula. } \\
\text { Genera buenos resultados en la } \\
\text { evaluación diagnóstica de las } \\
\text { competencias: matemática, } \\
\text { lingüística y expresión oral. }\end{array}$ \\
\hline $\begin{array}{l}\text { Pedreira y } \\
\text { González } \\
(2014)\end{array}$ & $\begin{array}{l}\text { Descriptivo- } \\
\text { cualitativo }\end{array}$ & $\begin{array}{l}\text { Alumnado de } 30 \text { y } 40 \\
\text { de primaria ( } n=19 \text {, de } \\
\text { ellos } 1 \text { con Trastorno } \\
\text { General del } \\
\text { Desarrollo) y } 2 \\
\text { profesoras. Se lleva a } \\
\text { cabo en ciencias } \\
\text { sociales y naturales. }\end{array}$ & $\begin{array}{l}\text { Cambia la participación del } \\
\text { alumnado, mejora el trabajo en } \\
\text { equipo, aumenta la atención y la } \\
\text { amistad. Todos se ayudan } \\
\text { existiendo una inclusión efectiva y } \\
\text { real entre los participantes. }\end{array}$ \\
\hline $\begin{array}{l}\text { Rodríguez } \\
\text { (2014) }\end{array}$ & Descriptivo & $\begin{array}{l}\text { Alumnado de } 20 \text { de } \\
\text { primaria ( } n= \\
\text { desconocido, no se } \\
\text { incluye el total). Se } \\
\text { lleva a cabo en el área } \\
\text { de matemáticas. }\end{array}$ & $\begin{array}{l}\text { Mejoran muchas de las } \\
\text { competencias del alumnado. } \\
\text { Sobre todo mejora la matemática. } \\
\text { Los alumnos muestran más } \\
\text { autonomía y responsabilidad. }\end{array}$ \\
\hline
\end{tabular}




\begin{tabular}{llll}
\hline \multicolumn{1}{c}{ Estudio } & \multicolumn{1}{c}{ Diseño } & Participantes/áreas & Resultados \\
\hline Iglesias y López & Cuasi- & Alumnado de 60 de & Se observa un clima adecuado de \\
(2014) & experimental & $\begin{array}{l}\text { primaria }(n=13) \text {. Se } \\
\text { aplica dentro del área }\end{array}$ & $\begin{array}{l}\text { convivencia en el aula. Los } \\
\text { alumnos están más motivados. }\end{array}$ \\
& de & Además, se facilita la adquisición y \\
& matemáticas. & desarrollo de habilidades sociales \\
& & y mejora el rendimiento \\
& & académico sobre todo el de \\
& & alumnos con mayores \\
& & dificultades.
\end{tabular}

\begin{tabular}{lll}
\hline González y & Descriptivo & Alumnado de 10 de \\
Aguaded (2015) & primaria $(n=14)$. Se \\
& desarrolla en las áreas \\
& de matemáticas y \\
& lengua castellana.
\end{tabular}

\begin{tabular}{lll}
\hline $\begin{array}{l}\text { Valdebenito y } \\
\text { Durán (2015) }\end{array}$ & $\begin{array}{l}\text { Cuasi- } \\
\text { experimental }\end{array}$ & $\begin{array}{l}\text { Alumnado de 2o a 5ㅇ } \\
\text { de primaria }(n=202) . \\
\text { Se aplica en lengua, } \\
\text { matemáticas, ciencias } \\
\text { sociales y naturales. }\end{array}$
\end{tabular}

Mejora la autonomía en la realización de tareas, aunque perdura la idea de competitividad y el trabajo individual. Mejora el ambiente del aula y el nivel académico del alumnado.

Valdebenito y Cuasi- $\quad$ Alumnado de 2ㅇ a 5을 hay mejoras en la comprensión lectora de todos los alumnos del grupo de intervención. La comprensión lectora se evalúa en las áreas de: lengua, matemáticas, ciencias sociales y naturales.

\begin{tabular}{llll}
\hline Carrasco et al. & Cuasi- & Alumnado de 3ㅇ y 40 & Mejora el clima del aula y la \\
(2015) & experimental & de primaria $(n=78)$. & conducta prosocial del alumnado.
\end{tabular}
Se da en ciencias naturales y sociales.

Flores y Duran Cuasi- Alumnado de 3으 a 6은 lectora
(2016) experimental de primaria $(n=577)$ en lengua española muestra Se lleva a cabo en el mejoras significativas en el grupo área de lengua. de intervención frente al grupo control.

Iglesias, López y Cuasi- Alumnado de 2으 de Ambos grupos piensan que las Fernández-Río experimental primaria $(n=33)$. Se clases son divertidas, pero se (2017) desarrolla en el área de matemáticas. destaca una percepción más positiva en el cooperativo. Además, este grupo alcanzó niveles más altos de rendimiento.

\begin{tabular}{lll}
\hline Mato-Vázquez, & Cuasi- & Alumnado de 60 de \\
Espiñeira y & experimental & $\begin{array}{l}\text { primaria }(n=149) . \text { Se } \\
\text { da en el área de } \\
\text { López-Chao }\end{array}$ \\
(2017) & & matemáticas.
\end{tabular}

Aumenta la atención y comprensión de problemas. Incrementa la autoconfianza y motivación. Los alumnos valoran positivamente trabajar de forma cooperativa.

\begin{tabular}{|c|c|c|c|}
\hline $\begin{array}{l}\text { Polo, Mendo, } \\
\text { Fajardo y León } \\
(2017)\end{array}$ & $\begin{array}{l}\text { Cuasi- } \\
\text { experimental }\end{array}$ & $\begin{array}{l}\text { Alumnado de } 50 \text { y } 60 \\
\text { de primaria }(n=110) \text {. } \\
\text { Se desarrolla en las } \\
\text { áreas de primaria. }\end{array}$ & $\begin{array}{l}\text { Mediante el aprendizaje } \\
\text { cooperativo se reducen las } \\
\text { agresiones verbales y físicas } \\
\text { directas, físicas indirectas y de } \\
\text { exclusión social. }\end{array}$ \\
\hline $\begin{array}{l}\text { Ribosa y Durán } \\
\text { (2017) }\end{array}$ & Descriptivo & $\begin{array}{l}\text { Alumnado de } 30,40 \text { y } \\
50 \text { de primaria } \\
\text { ( } n=24) \text {. Se aplica en } \\
\text { el área de } \\
\text { matemáticas. }\end{array}$ & $\begin{array}{l}\text { El aprendizaje cooperativo } \\
\text { fomenta la interacción entre } \\
\text { todos. Además, se promueven los } \\
\text { razonamientos matemáticos, el } \\
\text { pensamiento estratégico, la } \\
\text { adivinación y la planificación. }\end{array}$ \\
\hline Estudio & Diseño & Participantes/áreas & Resultados \\
\hline
\end{tabular}




\begin{tabular}{|c|c|c|c|}
\hline Yuste (2017) & $\begin{array}{c}\text { Cuasi- } \\
\text { experimental }\end{array}$ & $\begin{array}{l}\text { Alumnado de } 10,20 \text { y } \\
\text { 3o de primaria }(n= \\
\text { 110). Se desarrolla en } \\
\text { el área de } \\
\text { matemáticas. }\end{array}$ & $\begin{array}{l}\text { El alumno presenta conductas } \\
\text { más autónomas y espontáneas. } \\
\text { También aumenta la motivación } \\
\text { evidenciándose una actitud } \\
\text { entusiasta por el trabajo. }\end{array}$ \\
\hline $\begin{array}{l}\text { Iglesias, García- } \\
\text { Riaza y } \\
\text { Sánchez-Gómez } \\
\text { (2017) }\end{array}$ & $\begin{array}{c}\text { Cuasi- } \\
\text { experimental }\end{array}$ & $\begin{array}{c}\text { Alumnado de } 50 \text { de } \\
\text { primaria ( } \mathrm{n}=19 \text { ) y } 6 \\
\text { maestros. Se da en } \\
\text { lengua y se trabajan } \\
\text { contenidos concretos } \\
\text { de ciencias sociales, } \\
\text { naturales y } \\
\text { matemáticas. }\end{array}$ & $\begin{array}{l}\text { Los estudiantes tienden a mostrar } \\
\text { un alto grado de motivación y } \\
\text { satisfacción con el trabajo } \\
\text { cooperativo. Ahora, se relacionan } \\
\text { mejor con los compañeros de } \\
\text { clase y el trabajo en grupo ha } \\
\text { facilitado hacer la tarea con éxito. }\end{array}$ \\
\hline
\end{tabular}

Tabla 3. Descripción de los estudios sobre el aprendizaje cooperativo en las áreas troncales de primaria.

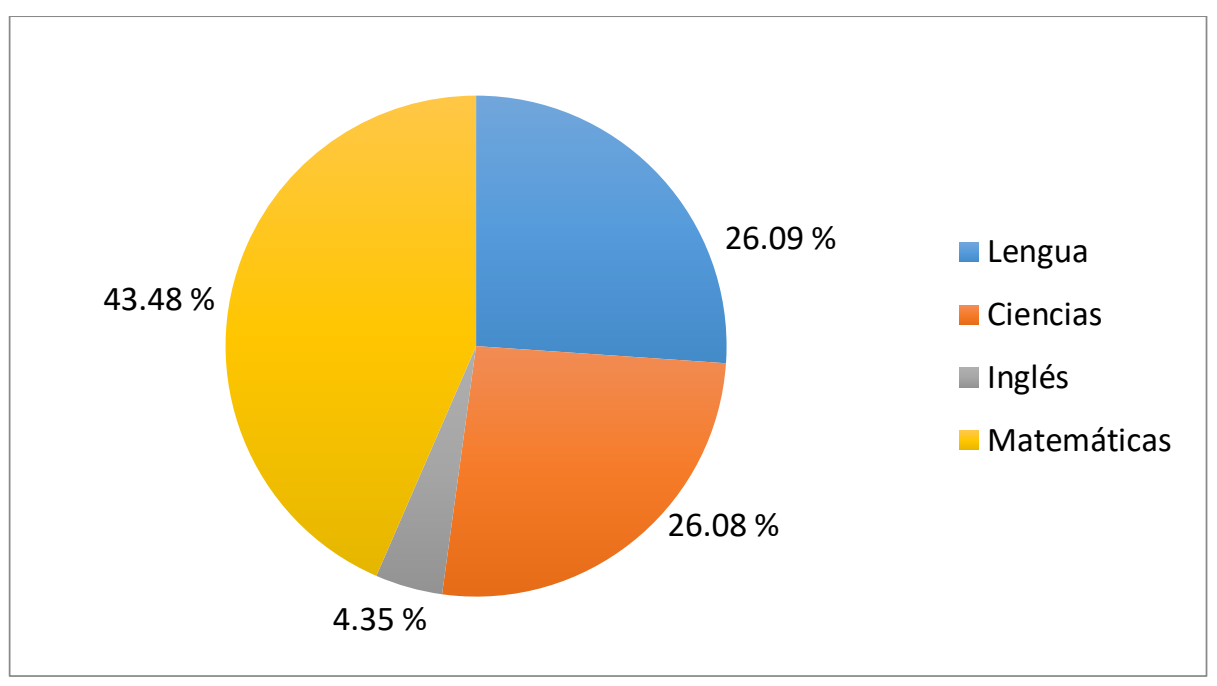

Figura 1. Porcentajes de aprendizaje cooperativo tras aplicar los criterios de inclusión/exclusión.

De los 14 trabajos analizados sobre el aprendizaje cooperativo, 7 (50\%) se centran en el estudio de solo una de las 5 áreas curriculares consideradas troncales, 6 (42.86\%) incluyen dos o más áreas troncales y 1 (7.14\%) aborda las áreas de la etapa de primaria de una manera global. Por tanto, un $92.86 \%$ de los trabajos de la Tabla 3 señalan sobre qué áreas troncales se emplea el aprendizaje cooperativo. En relación al área de lengua castellana y literatura se incluyen 6 trabajos (Flores y Duran, 2016; Gallach y Catalán, 2014; González y Aguaded, 2015; Iglesias, García-Riaza et al., 2017; Polo et al., 2017; Valdebenito y Durán, 2015). Respecto a matemáticas, se han registrado un total de 10 estudios (González y Aguaded, 2015; Iglesias, García-Riaza et al., 2017; Iglesias y López 2014; Iglesias, López et al., 2017; Mato-Vázquez et al., 2017; Polo et al., 2017; Ribosa y Durán, 2017; Rodríguez, 2014; Valdebenito y Durán, 2015; Yuste, 2017). El aprendizaje cooperativo se desarrolla en el área de inglés únicamente en el trabajo de Polo et al. (2017). Por último, las áreas de ciencias de la naturaleza y ciencias sociales comparten el mismo número de trabajos, 6 , ya que los autores de los mismos no han considerado la opción de centrarse exclusivamente en una de ellas (Carrasco et al., 2015, Gallach y Catalán, 2014; Iglesias, García-Riaza et al., 2017; Pedreira y González, 2014; Polo et al., 2017; Valdebenito y Durán, 2015). Véase Figura 1. 
En los trabajos analizados en la presente revisión, se observan un total de 29 beneficios. De ese total, el $31.03 \%$ se asocian a mejoras en las relaciones y habilidades sociales del alumnado (Carrasco et al. 2015; Flores y Duran, 2016; Gallach y Catalán, 2014; González y Aguaded, 2015; Iglesias, García-Riaza et al., 2017; Iglesias y López 2014; Pedreira y González, 2014; Polo et al., 2017; Ribosa y Durán, 2017). El 27.59\% de los beneficios se incluyen dentro del rendimiento académico y competencial del alumnado (Gallach y Catalán, 2014; González y Aguaded, 2015; Iglesias y López, 2014; Iglesias, López et al., 2017; Mato-Vázquez et al., 2017; Ribosa y Durán, 2017; Rodríguez, 2014; Valdebenito y Durán, 2015). El 17.24\% se corresponde con la motivación (Iglesias, García-Riaza et al., 2017; Iglesias y López 2014; Mato-Vázquez et al., 2017; Yuste, 2017). Otro $10.34 \%$ se detecta con el aumento de la autonomía del alumnado (González y Aguaded, 2015; Rodríguez, 2014; Yuste, 2017). Un 6.90\% de los beneficios encontrados tienen relación con un aumento de la atención (Mato-Vázquez et al., 2017; Pedreira y González, 2014). Por último, un $3.45 \%$ de los beneficios de aprendizaje cooperativo se corresponde con mejoras en la autoconfianza (Mato-Vázquez et al., 2017) y otro $3.45 \%$ con la responsabilidad (Rodríguez, 2014).

Respecto a los elementos negativos detectados en la revisión se hace alusión a tres aspectos. En primer lugar, el trabajo de González y Aguaded (2015) considera que a pesar de las mejoras que se detectan perdura el trabajo individual entre el alumnado. En segundo lugar, estos mismos autores añaden que la competitividad en clase no desaparece y el aprendizaje cooperativo se debería alejar de ese modelo competitivo e individualista. Por último, el trabajo de Carrasco et al. (2015) apunta que tras el empleo del aprendizaje cooperativo no disminuye significativamente la violencia sufrida ni la observada.

\section{Discusión}

Respecto a los beneficios sobre el rendimiento académico, el presente trabajo de revisión ha encontrado diferentes mejoras dentro del área de matemáticas. Concretamente, en las destrezas específicas de pensamiento estratégico y predictivo, planificación y experimentación (González y Aguaded, 2015; Iglesias y López, 2014; Iglesias, López et al., 2017; Ribosa y Durán, 2018; Rodríguez, 2014). Estos resultados podrían deberse a las características propias del área ya que muchas de las actividades permiten que los alumnos puedan ayudarse durante el aprendizaje de los conceptos más difíciles. Estas mejoras académicas en matemáticas coinciden con las obtenidas por Pons, González-Herrero y Serrano (2008), donde se confirma que las diferencias de rendimiento más significativas se dan en el trabajo con contenidos más complejos. Otra posible causa de esta mejora académica podría ser debida al aumento de la motivación hacía las matemáticas como consecuencia del trabajo en grupos (Iglesias, García et al., 2017; Iglesias y López, 2014; Mato-Vázquez, 2017; Yuste, 2017). Pese a encontrar, inicialmente, un porcentaje no muy elevado de publicaciones sobre la aplicación del aprendizaje cooperativo en matemáticas (17.27\%), sus efectos académicos parecen ser más importantes que en el resto de áreas troncales que trabajan contenidos más concretos y familiares los cuales supondrían una menor dificultad para los alumnos.

Cid (2017) recoge como beneficios de esta metodología: el aprendizaje de contenidos, en la mejora de la escritura, en la eficacia para la resolución de problemas, en la comprensión lectora y en la asimilación de conceptos científicos, sin embargo, la presente revisión encuentra escasas evidencias al respecto. Estas ausencias de resultados positivos sobre el rendimiento podrían deberse a las dificultades existentes para evaluar el rendimiento escolar de una manera objetiva controlando todos los factores individuales, familiares y escolares implicados (Santos-Rego, Godas y Lorenzo, 2012). Por ello, existen trabajos que tratan de valorar el rendimiento escolar a partir de otras variables como: los hábitos de estudio, la satisfacción con la materia, el apoyo familiar y las opiniones sobre la escuela, vinculadas con la mejora del rendimiento (Santos-Rego, 
Ferraces, Godas y Lorenzo, 2018). Los trabajos analizados en la presente revisión que se centran en el rendimiento académico analizan principalmente los cambios en la competencia matemática y en la competencia lingüística y comunicativa. La LOMCE (2013) y su nueva clasificación de las áreas en troncales, obligatorias y de libre configuración autonómica parece no haber cambiado la preocupación de los investigadores por evaluar el rendimiento de las áreas consideradas instrumentales: matemáticas y lengua castellana y literatura.

Los resultados recogidos en la revisión relativos a los aspectos afectivos y sociales son mucho más sólidos que los obtenidos sobre el rendimiento. En los trabajos analizados, se desprende que con la implementación del aprendizaje cooperativo en las áreas troncales de primaria se fortalecen las relaciones sociales, mejora la convivencia en el aula, se favorece a la conducta prosocial, se fomenta la responsabilidad y la amistad y aumenta la inclusión. En esta misma línea, el trabajo de Benítez (2017) también considera esta metodología adecuada para la inclusión en el aula ordinaria de alumnado con Síndrome de Down, independientemente de la etapa educativa. En definitiva, parece existir un consenso entre los investigadores sobre que el aprendizaje cooperativo activa la comunicación e interacción entre grupos heterogéneos suponiendo mayor cohesión, reduciendo el riesgo de comportamientos conflictivos y favoreciendo la inclusión escolar, la autonomía y la participación (Pedreira y González, 2014; Polo et al., 2017; Pujolàs, 2004).

En los últimos años es frecuente encontrar, en el contexto de la enseñanza de idiomas, numerosas referencias al aprendizaje cooperativo y a las posibilidades que este ofrece para trabajar una lengua extranjera en el aula (Ruiz-Martínez y Ruiz-Fernández, 2014). Sin embargo, tras aplicar los criterios de inclusión y exclusión el presente trabajo no incluye ningún artículo centrado en el inglés. Este hecho podría deberse a que los alumnos de primaria tienen mayores limitaciones a la hora de comunicarse y ayudarse entre ellos en inglés sin recurrir al castellano. Por tanto, quizás para el profesorado de lenguas extranjeras en secundaria y bachillerato tenga mayor utilidad como así reflejan algunos trabajos encontrados (Faya y Chao, 2015; Warburton, 2017). En primaria algunos de los trabajos centrados en el área de inglés como el de Carrión (2018) y Orcera et al. (2017) se excluyeron de esta revisión, ya que limitaban el planteamiento a prácticas en grupo que no tenían en cuenta el resto de aspectos básicos del aprendizaje cooperativo como el empleo de técnicas concretas que fomentan la interdependencia positiva, o la asunción de roles específicos dentro del grupo.

En relación al segundo objetivo, se plantean futuras líneas de actuación relativas al aprendizaje cooperativo en las áreas troncales de la etapa de primaria. Tras la revisión de los trabajos incluidos, los autores del presente trabajo consideran necesario un cambio en la relación actual familia-escuela. La bibliografía consultada coincide, entre las conclusiones de las experiencias docentes, en que la participación de las familias y su apoyo en la aplicación de técnicas cooperativas serán clave para la buena práctica en el aula (Carrasco et al., 2015). A nivel científico, sería interesante aumentar la presencia de las familias en más investigaciones educativas, como se expone en el trabajo de Carrasco et al. (2015), ya que pueden ser una fuente de información muy importante.

De cara a futuros estudios centrados en el aprendizaje cooperativo, los autores del presente trabajo insisten en la conveniencia de que los investigadores cuenten con muestras amplias. Además, se considera necesario que se describa adecuadamente la técnica utilizada y que tengan un tiempo de aplicación considerable para poder extraer resultados concluyentes, en la línea de las conclusiones de Iglesias, López et al. (2017). También se debe hacer hincapié en la necesidad de emplear cuestionarios validados para extraer evidencias más objetivas que las percepciones de profesores, alumnos y/o familias. 
Como propuesta de investigación futura, se plantea la opción de indagar en la formación docente en relación al aprendizaje cooperativo. Experiencias previas como la de Lara (2015) muestran la necesidad de avanzar en esta línea de estudio para mejorar la aplicación en las aulas de esta metodología de trabajo.

Por último, se consideran necesarias nuevas publicaciones que relacionen el aprendizaje cooperativo con las Tecnologías del Aprendizaje y el Conocimiento (TAC). Pese a que en la presente revisión no se han encontrado publicaciones en este sentido, los futuros investigadores deberían considerar la utilización y estudio de las TAC en el desarrollo de esta metodología. No se debe pasar por alto que la LOMCE (2013) considera las nuevas tecnologías como fundamentales para el cambio metodológico en el sistema educativo español al ser un elemento motivador y significativo en el aprendizaje del alumnado.

\section{Conclusiones}

La revisión bibliográfica realizada muestra que el aprendizaje cooperativo tiene una presencia desigual en las áreas troncales de primaria dentro del contexto español. En este sentido, destaca la utilización de esta metodología en matemáticas por delante de las áreas de lengua, ciencias e inglés, siendo esta última la que recibe una atención mucho menor.

Los beneficios afectivos y sociales que aporta el aprendizaje cooperativo se detectan desde cualquiera de las áreas troncales de primaria. También se observa que el aumento de las habilidades sociales y el fomento de las relaciones grupales mejoran el clima del aula.

En definitiva, con los datos que se desprenden del presente análisis, se puede considerar el aprendizaje cooperativo como una herramienta útil para el desarrollo de las habilidades sociales y afectivas del alumnado de primaria. No obstante, se precisan más investigaciones centradas sobre todo en el rendimiento académico del alumnado. Para ello, se insiste en la necesidad de emplear diseños de corte longitudinal, con muestras amplias y la presencia de un grupo control para llegar a conclusiones más sólidas sobre los beneficios del aprendizaje cooperativo.

\section{Referencias bibliográficas}

Benítez, A. M. (2017). Aprendizaje cooperativo: Metodología de impulso para la inclusión educativa del alumnado con síndrome de Down en el aula ordinaria. En E. López-Meneses, D. Cobos, A. H., Martín, L., Molina-García y A. Jaén (Eds.), Innovagogía 2016. III Congreso Internacional sobre Innovación Pedagógica y Praxis Educativa. Libro de Actas (pp. 56-64). Sevilla: AFOE Formación

Carrasco, C., Alarcón, R. y Trianes, M. V. (2015). Eficacia de una intervención psicoeducativa basada en clima social, violencia percibida y sociométricos en alumnado de educación primaria. Revista Psicodidáctica, 20(2), 247-262. doi: 10.1387/RevPsicodidact.13206

Carrión, E. (2018). Experiencias TIC en la enseñanza bilingüe mediante recursos digitales musicales. Didáctica, Innovación y Multimedia, 36, 1-18.

Cassany, D. (2009). Conferencia plenaria: Competencias comunicativas y diversidad, European Seminar "The development of communicative skills in teacher training for an inclusive school". Fundación SM, Universidad Complutense de Madrid, Madrid 16-1-2009.

Chamseddine, M. (2018). Polarización escolar en España. Retos e implicaciones. International Journal of Educational Research and Innovation, 9, 189-205. 
Cid, T. (2017). La intervención participante como experiencia didáctica inclusiva. En E. LópezMeneses, D. Cobos, A. H., Martín, L., Molina-García y A. Jaén (Eds.), Innovagogía 2016. III Congreso Internacional sobre Innovación Pedagógica y Praxis Educativa. Libro de Actas (pp. 320-326). Sevilla: AFOE Formación

Cohen, E. G. (1994). Le travail de groupe. Stratégies d'enseignement pour la clase hétérogène. Montréal: Chenelière Éducation.

DeVries, D. L. y Edwards, K. J. (1974). Students teams and learning games: Their effects on crossrace and cross-sex interaction. Journal of Educational Psychology, 66, 741-749.

Faya, F. y Chao, M. (2015). Board-games as review lessons in English language teaching: useful resources for any level. Docencia e Investigación: Revista de la Escuela Universitaria de Magisterio de Toledo, 25(2), 67-82.

Fathman, A. K. y Kessler, C. (1993). Cooperative language learning in school contexts. Annual Review of Applied Linguistics, 13, 127-42.

Fernández-Río, J. (2014). Aportaciones del modelo de responsabilidad personal y social al aprendizaje cooperativo. En C. Velázquez, J. Roanes y F. Vaquero (Eds.), Actas del IX Congreso Internacional de Actividades Físicas Cooperativas (pp. 69-83). Málaga: La peonza.

Flores, M. y Duran, D. (2016). Tutoría entre iguales y comprensión lectora: ¿un tándem eficaz? Los efectos de la tutoría entre iguales sobre la comprensión lectora. Universitas Psychologica, 15(2), 339-352. doi: http://dx.doi.org/ 10.11144/Javeriana.upsy15-2.teic

Gallach, M. J. y Catalán, J. P. (2014). Aprendizaje cooperativo en primaria: Teoría, práctica y actividades concretizadas. Didáctica de las Ciencias Experimentales y Sociales, 28, 109133. doi: $10.7203 /$ DCES. 28.3810

González-Mayorga, H., Vidal, J. y Vieira, M. J. (2017). El impacto del informe PISA en la sociedad española: el caso de la prensa escrita. Relieve, 23(1), 1-16. doi: http://doi.org/10.7203/relieve.23.1.9015

González, A. y Aguaded, E. (2015). Aprendizaje cooperativo como aprendizaje efectivo. ReiDoCrea, 4, 206- 212.

Iglesias, A., García-Riaza, B. y Sánchez-Gómez, M. C. (2017). Collaborative learning and mobile devices: An Educational experience in primary education. Computers in Human Behavior, 72, 664-677. doi: 10.1016/j.chb.2016.07.019

Iglesias, J. C. y López, T. (2014). Estudiar y aprender en equipos cooperativos: Aplicación de la técnica TELI (Trabajo en Equipo-Logro Individual) para trabajar contenidos matemáticos. Magister, 26, 25-33.

Iglesias, J. C., López, T. y Fernández-Río, J. (2017). La enseñanza de las matemáticas a través del aprendizaje cooperativo en $2 \circ$ curso de educación primaria. Contextos Educativos, 2, 4764. doi: http://doi.org/10.18172/con.2926.

Instituto Nacional de Evaluación Educativa (2016). TIMSS 2015. Estudio internacional de tendencias en matemáticas y ciencias. Informe español: resultados y contexto. Madrid: Secretaría General Técnica.

Instituto Nacional de Evaluación Educativa (2017). PIRLS 2016. Estudio internacional de progreso en comprensión lectora. Informe español. Madrid: Secretaría General Técnica.

Johnson, D.W. y Johnson, R. T. (1994). Aprender juntos y solos. Buenos Aires: Aique Grupo Editor. 
Kagan, S. y Kagan, M. (1994). The Structural Approach: Six keys to cooperative. En S. Sahran (Ed.), Handbook of Cooperative Learning methods (pp. 115-133). Wesport: Greenwood Press.

Lara, R. S. (2015). Las competencias y el aprendizaje cooperativo. Resultados de una experiencia de investigación con docentes de primaria. En AIDIPE (Ed.), Investigar con y para la sociedad (pp. 1045-1055). Cádiz: Bubok.

Lata, S. y Castro, M. M. (2016). El aprendizaje cooperativo, un camino hacia la inclusión educativa. Revista Complutense de Educación, 27(3), 1085-1101. doi: http://dx.doi.org/10.5209/rev_RCED.2016.v27.n3.47441

Ley Orgánica 2/2006, de 3 de mayo, de Educación (BOE, de 4 de mayo).

Ley Orgánica 8/2013, de 9 de diciembre, para la mejora de la calidad educativa (BOE, de 10 de diciembre).

Lobato, C. (1997). Hacia una comprensión del aprendizaje cooperativo. Revista de Psicodidáctica, 4, 59-76.

Mato-Vázquez, D., Espiñeira, E. y López-Chao, V. A. (2017). Impacto del uso de estrategias metacognitivas en la enseñanza de las matemáticas. Perfiles Educativos, 158, 91-111.

Mega, A. M. y Liesa, M. (2015). El juego cooperativo como método para favorecer la inclusión y el desarrollo de conductas prosociales. Educación y Diversidad, 9(1-2), 17-30.

Orcera, E., Moreno, E. y Risueño, J. J. (2017). Aplicación de las TAC en un entorno AICLE: una experiencia innovadora en Educación Primaria. Aula de Encuentro, 19(1), 143-162.

Orden ECD65/2015, de 21 de enero, por la que se describen las relaciones entre las competencias, los contenidos y los criterios de evaluación de la educación primaria, la educación secundaria obligatoria y el bachillerato (BOE, de 29 de enero).

Pedreira, M. y González, M. P. (2014). El aprendizaje cooperativo en un centro de primaria: Una experiencia inclusiva. Innovación Educativa, 24, 259-272.

Pegalajar, M. C. (2018). Formación en competencias en alumnado universitario de Educación Social mediante prácticas basadas en el aprendizaje cooperativo. Revista Complutense de Educación, 29(3), 829-845. doi: http://dx.doi.org /10.5209/RCED.53970

Polo, M. I., Mendo, S., Fajardo, F. y León, B. (2017). Una intervención en aprendizaje cooperativo sobre el perfil del observador en la dinámica bullying. Universitas Psychologica, 16(1), 113. doi: http://dx.doi.org/10.11144/Javeriana.upsy16-1.iacp

Pons, R. M., González-Herrero, M. L. y Serrano, J. M. (2008). Aprendizaje cooperativo en matemáticas. Un estudio intracontenido. Anales de Psicología, 24(2), 253-261.

Pujolàs, P. (2004). Aprender juntos alumnos diferentes. Los equipos de aprendizaje cooperativo en el aula. Barcelona: Eumo-Octaedro.

Pujolàs, P. (2009). La calidad en equipos de aprendizaje cooperativo. Algunas consideraciones para el cálculo del grado de cooperatividad. Revista de Educación, 349, 225-239.

Pujolàs P. (2012). Aulas inclusivas y aprendizaje cooperativo. Educatio Siglo XXI, 30(1), 89-112.

Ribosa, J. y Durán, D. (2017). Cooperación, juego y matemáticas: Análisis de la aplicación del Tridio cooperativo con alumnado de primaria. PNA. Revista de Investigación en Didáctica de la Matemática, 11(3), 205-231.

Rodríguez, E. (2014). Los enigmas del Ogro de Halloween. Números. Revista de Didáctica de las Matemáticas, 85, 157-178. 
Ruiz-Martínez, L. y Ruiz-Fernández, J. M. (2014). Dinámicas de grupo, agrupamientos y trabajo cooperativo. En P. J. Molina-Muñoz (Ed.), Actas de las VI Jornadas de Formación para Profesores de Español en Chipre (pp. 43-58). Nicosia: ELEChipre.

Santos-Rego, M. A., Ferraces, M. J., Godas, A. y Lorenzo, M. D. M. (2018). Doo coperative learning and family involvement improve variables linked to academic performance? Psicothema,30(2), 212-217. doi: 10.7334/psicothema 2017.311

Santos-Rego, M. A., Godás, A. y Lorenzo, M. (2012). El perfil del alumnado repetidor y no repetidor en una muestra de estudiantes españoles y latinoamericanos: un estudio sobre los determinantes académicos. Estudios sobre Educación (ESE), 23, 43-62.

Santos-Rego, M. A., Lorenzo-Moledo, M. y Priegue-Caamaño, D. (2009). Aprendizaje cooperativo: práctica pedagógica para el desarrollo escolar y cultural. Magis. Revista Internacional de Investigación en Educación, 1(2), 289-303.

Slavin, R. E. (1980). Cooperative learning. Review of Educational Research, 1(2), 315-325.

Suárez, Z. (2013). El aprendizaje cooperativo. Una metodología para el mejoramiento del aprendizaje en el aula. Revista Calidad en la Educación Superior, 4(1), 26-46.

Valdebenito, V. y Durán, D. (2015). Formas de interacción implicadas en la promoción de estrategias de comprensión lectora a través de un programa de tutoría entre iguales. Revista Latinoamericana de Psicología, 47(2), 75-85.

Vázquez, S., Liesa, M. y Lozano, A. (2017). Recreos cooperativos e inclusivos a través de la metodología de Aprendizaje-Servicio. Revista Electrónica Interuniversitaria de Formación del Profesorado, 20(1), 173-185. doi: http://dx.doi.org/10.6018/reifop.20.1.213181

Warburton, S. (2017). Metodologías activas. Un estudio de AICLE/CLIL en Tecnología e Inglés. Tejuelo, 26, 213-238. doi: 10.17398/1988-8430.26.213

Yuste, E. M. (2017). Influencia de la introducción y desempeño de roles sobre las conductas interactivas del alumnado de Educación Primaria en un contexto de aprendizaje cooperativo (Tesis doctoral). Universidad de Murcia, Murcia. 